\title{
Probing the potential of structured and surface functionalized tools for dry cold forging of aluminium
}

\author{
Marco Teller ${ }^{1, *}$, Ingo Ross ${ }^{2}$, Stephan Prünte ${ }^{3}$, André Temmler ${ }^{4}$, Moritz Küpper $^{4}$, Reinhart Poprawe $^{2,4}$, \\ Jochen M. Schneider ${ }^{3}$, and Gerhard Hirt $^{1}$ \\ ${ }^{1}$ Institute of Metal Forming (IBF), RWTH Aachen University, Intzestr. 10, 52072 Aachen, Germany \\ ${ }^{2}$ Fraunhofer Institute for Laser Technology (ILT), Steinbachstr. 15, 52074 Aachen, Germany \\ ${ }^{3}$ Materials Chemistry (MCh), RWTH Aachen University, Kopernikusstr. 10, 52074 Aachen, Germany \\ ${ }^{4}$ Chair for Laser Technology (LLT), RWTH Aachen University, Steinbachstr. 15, 52074 Aachen, Germany
}

\begin{abstract}
In order to reduce friction and wear in cold forging of aluminium and its alloys a tremendous amount of lubricants is used. The main task of the liquid lubricants is the separation of tool and workpiece surfaces to avoid adhesion and abrasion. From an ecological and economical point of view, a reduction of the consumption of lubricants is meaningful. Novel surface modification techniques have been investigated to enable dry cold forming. In previous work, a reduced wear by adhesion of aluminium to an AISI H11 ESR tool steel surface was detected for a combination of laser polishing and a subsequent surface functionalization. Here, structured surfaces combined with surface functionalization are investigated regarding their wear behaviour. Therefore, different structures are realized on AISI H11 ESR tool steel surfaces by laser structuring. These structures are aligned parallel and perpendicular to the material flow direction. Furthermore, different structure depths are generated. It is evident that the laser induced surface melting causes both, a reduced micro roughness as well as a homogenized elementary distribution. Moreover, the surfaces are functionalized with an octadecylphosphonic acid selfassembled monolayer (C18PA-SAM). The SAM's distal alkyl termini functionalize the tool steel surface und thus only weak van-der-Waals interactions are present in the aluminium-tool steel interface rendering an interaction, which is expected to be similar to the interaction in conventionally lubricated aluminium forming. Finally, the surfaces are appraised regarding their wear behaviour in a compression-torsion-wear tribometer. The different experimental results document the potential of the here investigated combined tool surface modification. Although, the performance of a lubricated process is not completely reached a reduction in friction and wear can be achieved compared to unmodified tools.
\end{abstract}

Keywords: Tribology, cold forming, aluminium

\section{Introduction}

Tribological phenomena between tool and workpiece can significantly influence metal forming processes. For example, friction effects the material flow in the manufacturing process and due to wear of the tool surfaces, the boundary conditions of the process can change. In order to control friction and wear and to guarantee a stable production lubricants are widely used. Especially in cold forging, a high effort is spent to several process steps for lubricant application, subsequent removal and recycling or disposal, respectively [1].

Current research activities aim for a significant reduction of the lubricant usage culminating in dry metal forming [1]. Ecological and economic reasons are driving forces for the development of novel surface treatments and modifications to realize this purpose.

Prior to the application of new tribological solutions to the manufacturing process an extensive testing and characterization is done on lab-scale. Tribometers reproduce specific features of the real process and are widely used for a preliminary evaluation of tribologically optimized surfaces [2].

In previous research works, the authors documented the positive effect of laser polishing [3], a surface functionalization with self-assembled monolayers (SAM) $[4,5]$ and the combination of both strategies [6] for the reduction of aluminium adhesion and friction in a compression-torsion-wear tribometer simulating the load case in cold forging. Especially dry cold forging of aluminium is challenging due to the severe load case including high relative normal stresses and a significant surface expansion [7]. Both leading to a break-up of the passivating oxide layer and a direct contact between virgin aluminium and the tool surface [8]. The highly reactive behaviour of aluminium is documented in several papers, e.g. by Bolvardi et al. via ab initio calculations $[9,10]$ and experimentally by Fuentes et al. [11].

In this paper, another surface modification concept in order to reduce friction and wear is presented. Instead of a laser polishing treatment, which can decrease the micro and macro roughness, surface structuring by laser

\footnotetext{
* Corresponding author: teller@,ibf.rwth-aachen.de
} 
remelting is used on specimens from AISI H11 ESR in order to adjust the surface structure and to prepare it for a functionalization with self-assembled monolayers.

The paper is structured as follows: First, the specimen geometry, the laser structuring process for several surface structures and the subsequent functionalization step are described. Afterwards, results from the tribometer experiments are presented and discussed. The results show a positive effect of a circular laser structure with $1 \mu \mathrm{m}$ depth combined with SAM. The experiments show a reduced interaction between tool and workpiece.

\section{Specimen preparation}

\subsection{Tribometer specimens}

The wear behaviour of the different surface modifications is tested in a compression-torsion-wear tribometer. The specimen geometry is shown in Fig. 1.
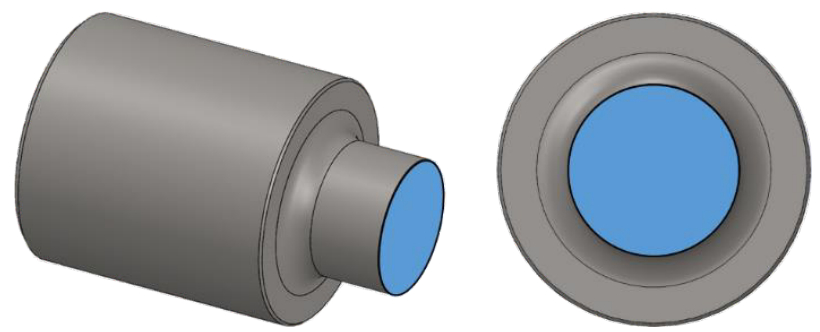

Fig. 1. Tool specimen for the compression-torsion-wear test with marked contact surface (blue) for the structuring process in side and top view.

The compression-torsion-wear tribometer reproduces main features of the cold forging process like a high relative contact pressure. The presented tool specimen from steel will be pressed on a workpiece specimen from pure aluminium. The workpiece specimen enables a surface expansion and as a result the break-up of the oxide layer. The work presented in [12] depicts further details regarding the compression-torsion-wear tribometer.

\subsection{Laser structuring}

Surface structuring by laser remelting is based upon the physical interrelationship between a modulation of melt pool volume and the dependent movement of the threephase line that determines the resulting surface topography while the molten material solidifies. In laser polishing a change of the melt pool volume is undesirable $[13,14]$. Surface structuring by remelting however, aims for a precise modulation of the melt pool volume, e.g. by a modulation of the laser power. As a consequence of the induced modulation of melt pool volume, the resulting surface topography is generated by the same mechanism as shown for ripple formation during laser polishing [15]. Figure 2 shows the process principle of surface structuring by laser remelting. By moving the laser beam relatively to the workpiece surface, a thin surface layer $(<100 \mu \mathrm{m})$ is molten. After the laser beam has passed, the material solidifies. The direction of the solidifaction is defined by the melt pool surface. At constant laser power, as used for
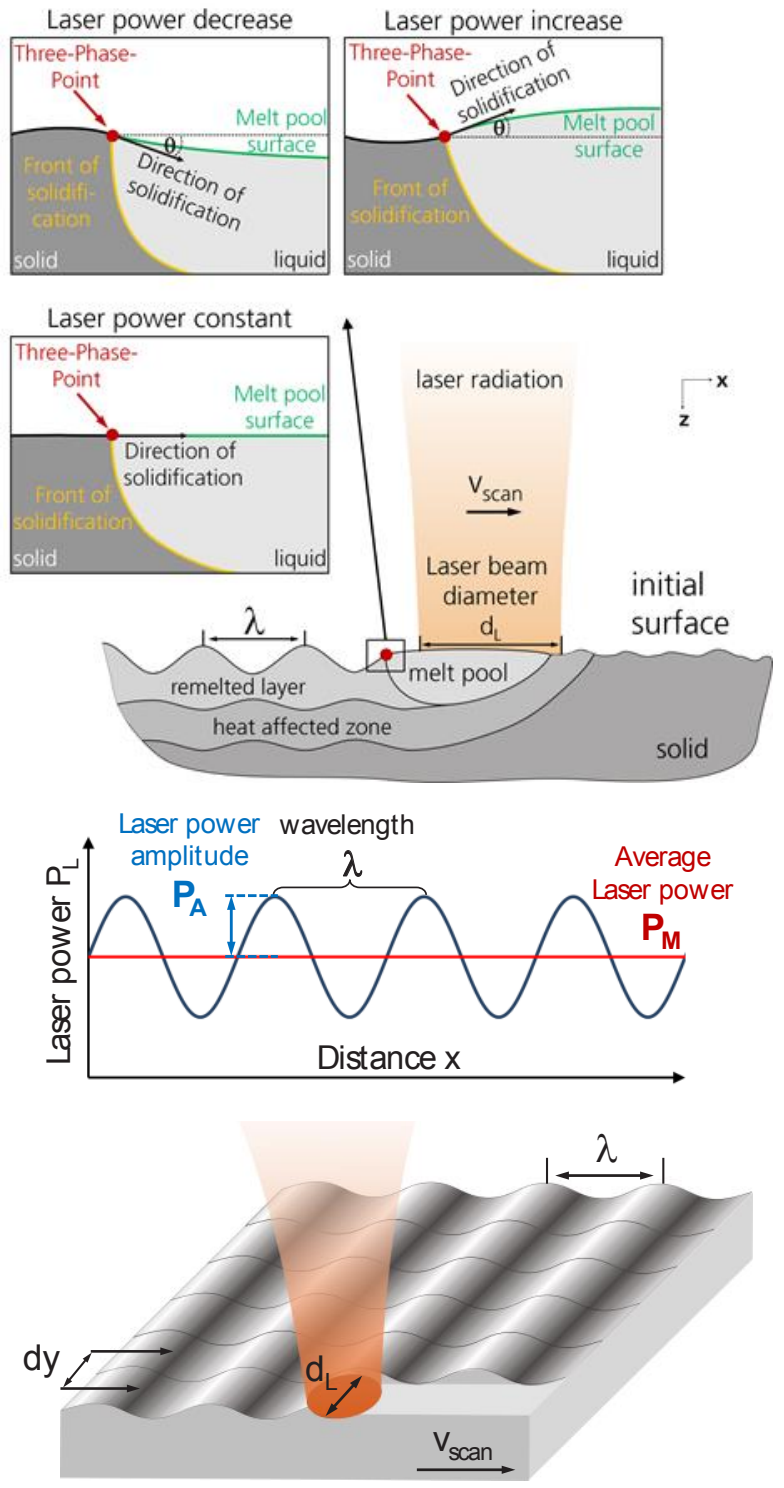

Fig. 2. Top: Schematics of the active principle for surface structuring by laser remelting; Bottom: Schematics of the process principle. [17]

laser polishing [14], the melt pool volume of the steady state process does not change, resulting in an approximately flat melt pool surface. Thus, no structuring occurs. Increasing the laser power will increase the amount of molten material and, due to density changes and thermal expansion, will lead to a bigger melt pool volume. The melt pool surface bulges outwards (see Fig. 2). Following the bulged surface, the material solidifies in an upwards-slope at the three-phase-point. Decreasing the laser power will lead to the opposite effect. Thus, structuring can be achieved by modulation of the melt pool volume. The precise control of the spatial and temporal deposition of the laser energy is essential for structuring by remelting. Height, size and form of the produced surface topography depend directly on the average melt pool volume and on the absolute change and time-dependent alteration rate of the melt pool volume. In order to achieve periodic structures, the laser power is modulated sinusoidal at an average laser power $\mathrm{P}_{\mathrm{M}}$ with an amplitude $\mathrm{P}_{\mathrm{A}}$ and a wavelength $\lambda$ (Fig. 2 middle). In 
contrast to the Surfi-Sculpt process, which takes advantage of the keyhole welding effect and uses both vapour pressure and surface tension variation for creating singular structures [16], surface structuring by laser remelting stays in the regime of heat conduction welding and allows areal modification of surfaces with smooth transitions between the periodical features and without evaporation of material.

The tribometer specimens are processed on the "POLAR" 5-axis laser polishing machine (Fig. 3). The integrated 3D galvanometric laser scanner deflects the laser beam on the workpiece and moves the laser spot at a defined scanning velocity $v_{\text {scan }}$ and track offset dy. The wavelength of the created structures is a function of the wavelength $\lambda$ of the laser power modulation and the scanning velocity $\mathrm{v}_{\text {scan }}$. Areal structures can be obtained by overlapping the remelted tracks (Fig. 2 bottom) [17].

Tribometer specimens investigated in this work are made from AISI H11 ESR and are structured with circular and star-shaped patterns by surface structuring by laser remelting (wave shape). During the tribometer test, the circular structures are parallel to the material flow and the star-shaped structures are perpendicular to the material flow. For structuring, a focus diameter $d_{L}=125 \mu \mathrm{m}$, scan velocity $v_{\text {scan }}=206 \mathrm{~mm} / \mathrm{s}$, average laser power $P_{M}=50 \mathrm{~W}$ and amplitude $\mathrm{P}_{\mathrm{A}}=5 \mathrm{~W}$ is used. For the circular patterns $\left(\lambda_{\text {circular }}=328 \mu \mathrm{m}\right)$ an oscillating process strategy is used where each remelting scan vector is turned by $1^{\circ}$. The star-shaped patterns consist of 100 modulation cycles, resulting in a radius-dependent wavelength between $\lambda_{\text {star, } \mathrm{r}=1 \mathrm{~mm}}=63 \mu \mathrm{m}$ and $\lambda_{\text {star }, \mathrm{r}=4.5 \mathrm{~mm}}=283 \mu \mathrm{m}$. This pattern is applied with a circular strategy with a track offset $\mathrm{dy}=20.5 \mu \mathrm{m}$. By changing the number of processing steps, specimens with average structure heights of $\mathrm{h}_{\text {structure }}=1 \mu \mathrm{m}$ and $10 \mu \mathrm{m}$ are realized.

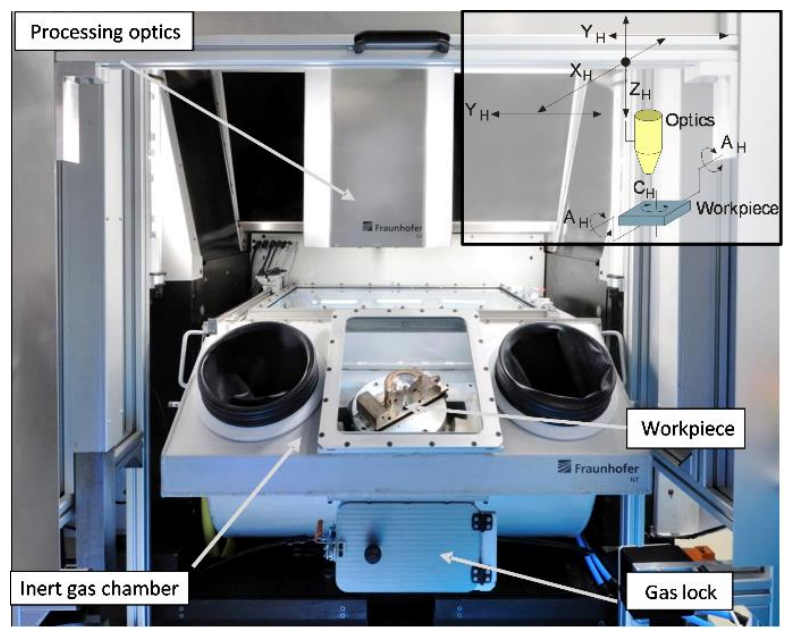

Fig. 3. Fraunhofer ILT's "POLAR" 5-axis laser polishing machine suitable for structuring by laser remelting. [18]

\subsection{Surface functionalization}

Octadecylphoshonic acid (C18PA) are deposited on laser structured surfaces of AISI H11 ESR by vacuum thermal evaporation at residual pressures of $5 \cdot 10^{-5} \mathrm{~Pa}$, followed by an annealing of the processed surface at $160{ }^{\circ} \mathrm{C}$. Reactant molecules are anchored to the tool surface forming P-O-
M ( $\mathrm{M}=$ Metal) bonds between the phosphonic acid headgroup and the oxide layer of the surface [19]. Henceforth, the surface is functionalized with a dense self-assembled monolayer (SAM) consisting of C18PA molecules having their alkyl moieties oriented distally to the tool surface. Previously, we showed that functionalization in such fashion lubricates the tool-workpiece interface and reduces $\mathrm{Al}$-adhesion on the tool surface due to acting vander-Waals interactions between alkyl moieties and the aluminium workpiece [4]. Contrarily, atomic scale onset of $\mathrm{Al}$-adhesion was observed in interfaces of aluminium and tool coatings in theory [9] and experiment [11]. Abinitio DFT calculations suggest the adsorption energies of Al-atoms to be two orders of magnitude higher in the latter interface than for interfaces with only van-derWaals interaction present [10].
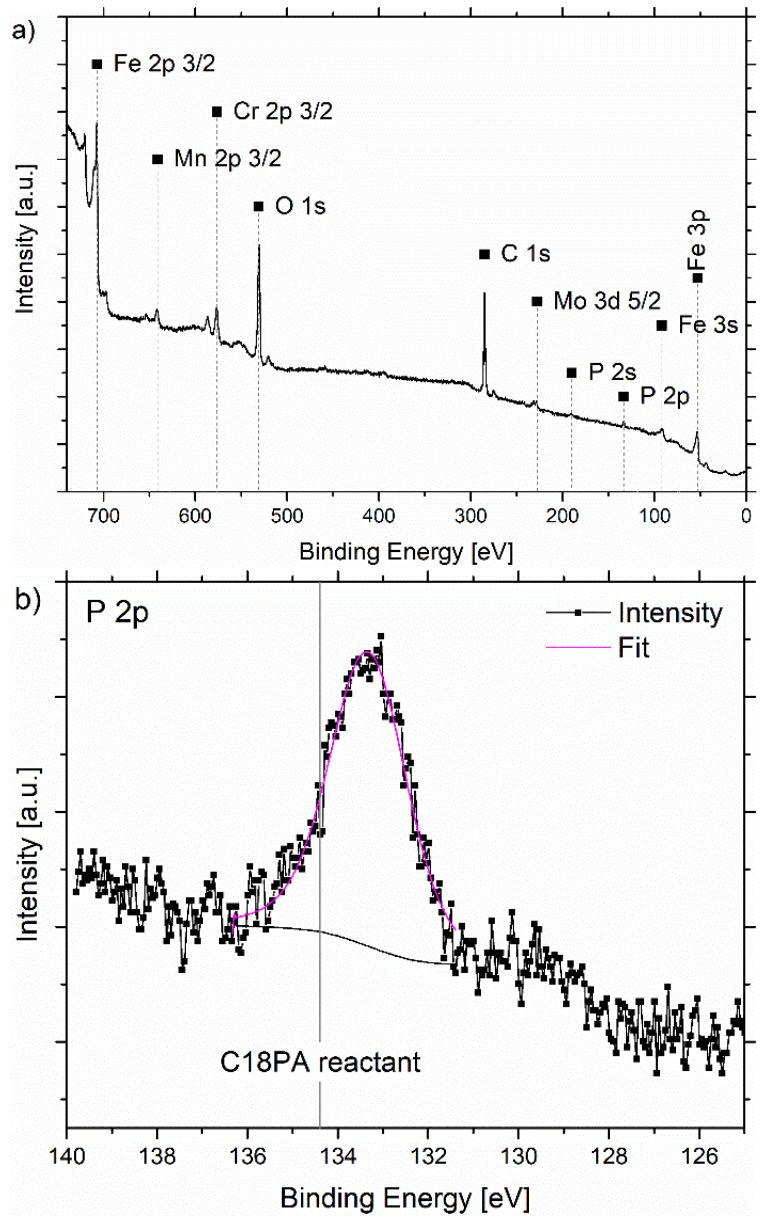

Fig. 4: (a) overview and (b) P 2p spectra obtained by XPS of $1 \mu \mathrm{m}$ circular laser structured surface functionalized with a C18PA-SAM.

Functionalized surfaces were investigated by X-ray photoelectron spectroscopy using a JEOL JAMP 9500F equipped with a non-monochromatic $\mathrm{Al} \mathrm{K} \alpha \mathrm{X}$-ray source. Acquired spectra were recorded with a step-size of $0.25 \mathrm{eV}$ and 5 measuring cycles for overview and $0.05 \mathrm{eV}$ and 20 cycles for detailed spectra, respectively while the pass-energy of the detector was set to $20 \mathrm{eV}$. Spectra analysis was carried out using the CASA XPS software. XPS-obtained surface chemistry of the $1 \mu \mathrm{m}$ circular structured tool discloses the presence of $\mathrm{P}$ beside $\mathrm{M}$ oxides on the surface originating from functionalization 
(Fig. 4a). The detected P $2 \mathrm{p}$ signal located at binding energies of $133.3 \mathrm{eV}$ (Fig. 4b) reveals an off-set of $1.1 \mathrm{eV}$ to the $\mathrm{P} 2 \mathrm{p}$ signal detected for $\mathrm{C} 18 \mathrm{PA}$ reactant indicative for formation of P-O-M bonds [4]. Thus, the surface of laser structured AISI H11 ESR tool steel is functionalized with C18PA-SAM anchored by P-O-M bonds, while vander-Waals interactions mediate the tool-workpiece due to distal alkyl moieties of the C18PA-SAM.

\section{Results}

The different modified tool surfaces were tested in the compression-torsion-wear tribometer at a normal pressure of three times the initial yield stress of pure aluminium for two rotations at a rotation velocity of one rotation per second. The normal pressure is less than in a typical cold forging process in order to allow for a more significant validation of the influence of the different surface modifications on tribological parameters.

The following sections present the transferred torque between tool and workpiece specimen, the surface characteristics measured via white light interferometry (WLI) before and after the wear test and the adhered aluminium volume on the tool specimen. These parameters allow for an assessment of the different surface modifications.

\subsection{Torque curves}

Figure 5 shows torque curves for the different structures perpendicular to the material flow direction. The starshaped structure leads to an oscillating shape with the same frequency as the rotational velocity of the tribometer. The deeper structure $\left(\mathrm{h}_{\text {structure }}=10 \mu \mathrm{m}\right)$ without functionalization generates the highest maximum torque $\mathrm{M}$ of approximately $30 \mathrm{Nm}$. The functionalization on the same structure reduces the maximum torque to circa $24 \mathrm{Nm}$. Moreover, the peak-to-valley value is less. The smooth structure with $\mathrm{h}_{\text {structure }}=1 \mu \mathrm{m}$ depth reaches approximately $26 \mathrm{Nm}$. The minimum value $(17 \mathrm{Nm})$ is found for the smooth structure combined with functionalization. Nevertheless, the functionalized surfaces show an increase for each peak value. This could be an indicator for an ongoing failure of the SAM layer. Figure 6 presents the torque curves for the different structures circular to the material flow direction. The several surfaces are determined in the same order as the star-shaped structures. The maximum torque values are found for the non-functionalized deep structure $(22 \mathrm{Nm})$ and the non-functionalized smooth structure $(22 \mathrm{Nm})$ followed by the functionalized deep structure $(15 \mathrm{Nm})$ and the functionalized smooth structure $(9 \mathrm{Nm})$. Again, an increase of the transferred torque for each peak can be detected for the SAM specimens. Moreover, the functionalized specimens show a more pronounced oscillation compared to the non-functionalized. Nevertheless, the circular structuring reveals a lower torque than for the star-shaped structure. Hence, the alignment of the structure to the material flow direction has a significant influence.

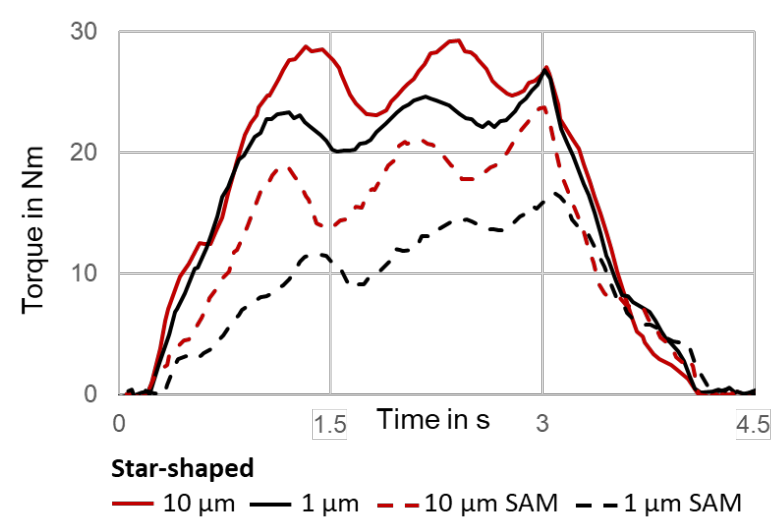

Fig. 5. Torque curves for star-shaped structures with different structure depths and functionalization.

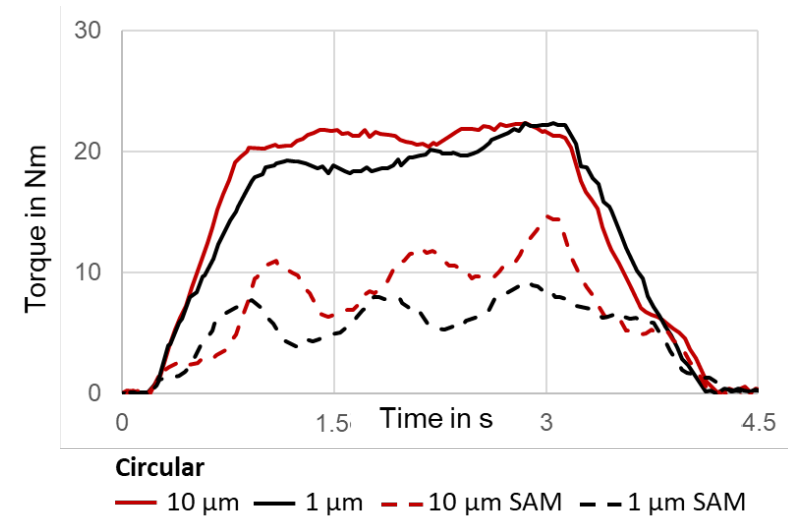

Fig. 6. Torque curves for circular structures with different structure depths and functionalization.

\subsection{WLI surfaces}

The surfaces of the tribometer specimens are measured with a Zygo NewView 7300 white light interferometer. Due to the known boundary effects at the specimens' outer radius [12], only the surface within an inner radius of $5 \mathrm{~mm}$ is evaluated. Figure 7 shows the resulting contact surfaces of the different surfaces with and without SAM coating after the test. The laser structured specimens with non-functionalized deep structures $\left(\mathrm{h}_{\text {structure }}=10 \mu \mathrm{m}\right)$, circular and star-shaped, show a significant amount of adhered aluminium on the surface. However, the distribution of the adhered material differs. For the circular structure, the material agglomerates to several larger lumps, which do not seem to have a preferred position on the underlying structure (Fig. 7 A2). For the star-shaped structures however, the areas with adhered material are distributed more evenly over the whole specimen and can be found predominately at the top of the structures and smearing down to the bottom (Fig. 7 A1). Taking the measured torque into account, these wear characteristics imply an increased tendency to shearing off aluminium for the star-shaped structure.

The specimens with functionalized deep structures show a significant reduction of adhered material. This is reflected in the reduced torque measured during the test for the coated specimens (Fig. 5 and 6). The amount of 
adhered aluminium is reduced for both structure types and the characteristics of the distribution is analogue to the non-functionalized specimens: on the one hand, scattered, albeit larger agglomerations for the circular structures, on the other hand, adhesions beginning the top and smearing to the bottom of the star-shaped structures.

This general behaviour can also be found on the specimens with smooth structures $\left(h_{\text {structure }}=1 \mu \mathrm{m}\right)$. While the amount of adhered material is, compared to the deeper structures, already reduced for the nonfunctionalized specimens, the SAM functionalization further decreases the volume of the adhered material. However, this decrease is less pronounced for the starshaped structure.
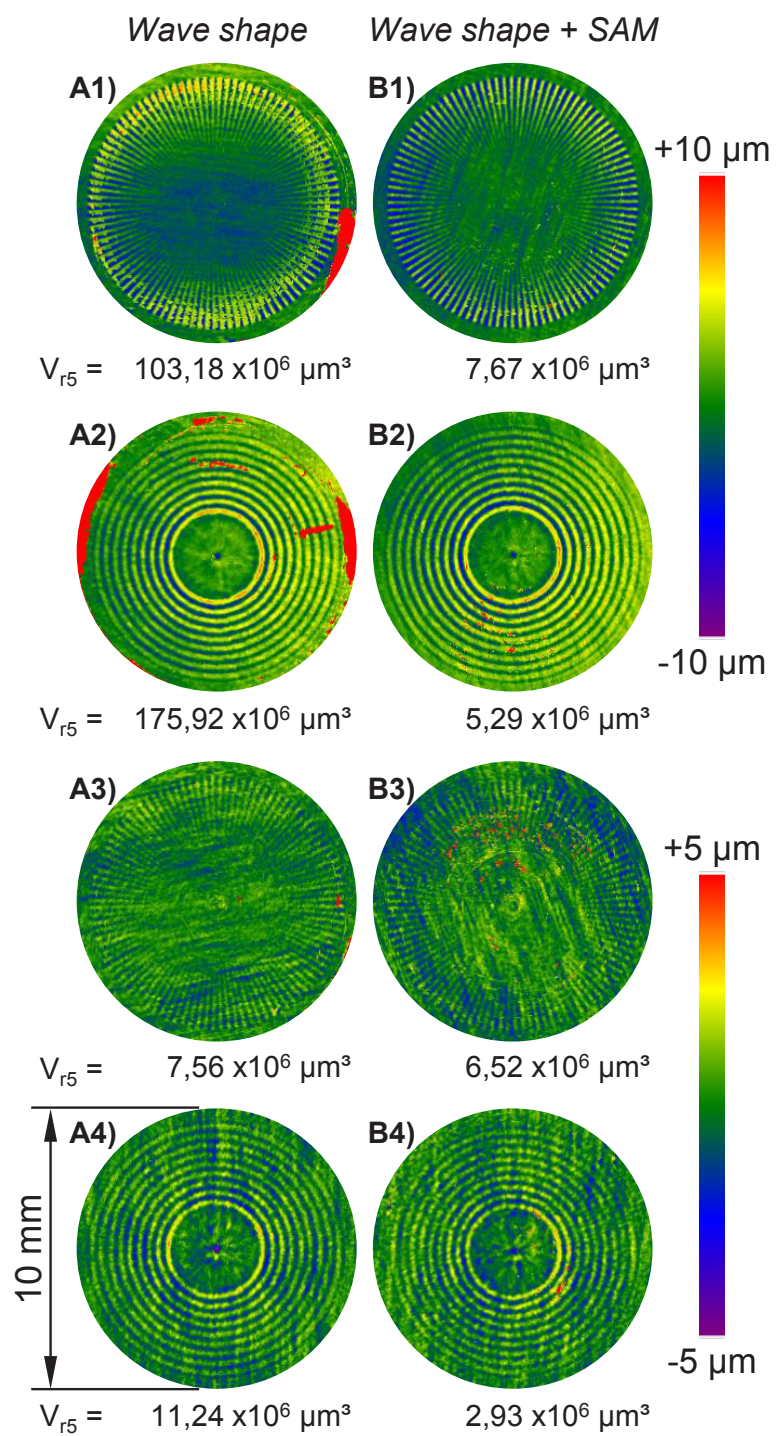

Fig. 7. WLI measurements of the overall adhered $\mathrm{Al}$ volume of different surfaces after testing in the tribometer. Columns: A) structured (Wave shape); B) structured and functionalized; Rows: 1) deep star-shaped structure (10 $\mu \mathrm{m}) ; 2)$ deep circular structure $(10 \mu \mathrm{m}) ; 3)$ smooth star-shaped structure $(1 \mu \mathrm{m}) ; 4)$ smooth circular structure $(1 \mu \mathrm{m})$

On the surface of the functionalized smooth star-shaped specimen, the above described adhesion pattern as well as several small agglomerations can be found (Fig. 7 B3). Similar agglomerations can also be found on the functionalized deep circular structure and may hint at a local failure of the SAM functionalization. This kind of wear characteristics cannot be detected on the functionalized smooth circular specimen (Fig. 7 B4). In addition, the circular specimen produces the smallest torque during the test and shows the smallest amount of adhered material. This leads to the hypothesis that the SAM functionalization did not fail under these specific load conditions.

\subsection{Adhered volumes}

By subtracting the surface topography of the unused specimen from the topography after the tribometer test, the volume of the adhered aluminium can be measured. This procedure was described in detail in [3].

A summary of the torque and adhered volume is given in Table 1 . The analysis of the adhered volume shows that the deep structure lead to the largest amount of adhered material. A possible explanation may be the increased shear load in the aluminium workpiece induced by the deep structures and a hypothetical increase of contact with non-oxidized aluminium due to the penetration of the structures into the natural oxide layer. Reducing the structure height as well as choosing a structure, which is parallel to the material flow results in a reduced amount of adhered aluminium. For all tested surfaces, the SAM functionalization is effectively reducing the adhered volume and measured torque. However, the reduction of the measured torque is less significant for the deep than for the smooth structures.

The smallest volume was measured for the functionalized smooth circular structure: $\mathrm{V}_{\text {circular, } 1 \mu \mathrm{m}, \mathrm{SAM}}=2.93 \times 10^{6} \mu \mathrm{m}^{3}$, which is, an almost $75 \%$ decrease in adhesive volume compared to the same surface without the C18PA-SAM. $\mathrm{V}_{\text {circular, } 1 \mu \mathrm{m}, \mathrm{SAM}}$ is slightly higher than the best result on a functionalized manually polished surface with $\mathrm{V}_{\text {polished,SAM }}$ $=2.11 \times 10^{6} \mu \mathrm{m}^{3}$ [5]. However, there is a greater than 2fold reduction in $\mathrm{M}_{\text {circular,1 } 1 \mathrm{~m}, \mathrm{SAM}}$ compared to both, the $1 \mu \mathrm{m}$, circular structured specimen without SAM and the manually polished surface with SAM, supporting the notion that combined functionalization with SAM and surface structuring decreases the overall process load in a real extrusion process.

Tab. 1. Maximum torque $\mathrm{M}$ and adhered aluminium volume $\mathrm{V}$ for different tested specimens

\begin{tabular}{lcc} 
Specimen & $\begin{array}{c}\text { Torque M } \\
{[\mathrm{Nm}]}\end{array}$ & $\begin{array}{c}\text { Volume V } \\
{\left[\mathrm{x} 10^{6} \mu \mathrm{m}^{3}\right]}\end{array}$ \\
\hline Manually polished* & $38^{*}$ & $5.12^{*}$ \\
Manually polished + SAM* & $20^{*}$ & $2.11^{*}$ \\
Circular $1 \mu \mathrm{m}$ & 22 & 11.24 \\
Circular $1 \mu \mathrm{m}+\mathrm{SAM}$ & 9 & 2.93 \\
Circular $10 \mu \mathrm{m}$ & 22 & 175.92 \\
Circular $10 \mu \mathrm{m}+\mathrm{SAM}$ & 15 & 5.29 \\
Star-shaped $1 \mu \mathrm{m}$ & 26 & 7.56 \\
Star-shaped $1 \mu \mathrm{m}+\mathrm{SAM}$ & 17 & 6.52 \\
Star-shaped $10 \mu \mathrm{m}$ & 30 & 103.18 \\
Star-shaped $10 \mu \mathrm{m}+\mathrm{SAM}$ & 24 & 7.67
\end{tabular}

*published by the authors in [5] 


\subsection{EDX measurements}

Additionally, the two SAM-functionalized specimens with $1 \mu \mathrm{m}$ structures, star-shaped and circular, respectively, were investigated by scanning electron microscopy (SEM) and energy-dispersive X-ray spectroscopy (EDX) after tribological experiments to characterize adhesion using a Zeiss $\Sigma$ igma SEM equipped with an Oxford Instruments X-MaxN 50 EDX-detector.

Fig. 8 depicts SE-micrographs of aluminium adhesion on star-shaped and circular structures, respectively, identified by EDX (Tab. 2). All investigated areas are localized $3 \mathrm{~mm}$ off the centre of each specimen, in the middle of the structured zone. Obviously, $\mathrm{Al}$ is attached to a larger degree on the star-shaped structures than on the circular surface (please note the factor 2-fold larger magnification necessary to show an overview of Aladhesion on the circular surface). Beside the macroscopic star-shaped or circular structure of the surface, a substructure is visible which is circular for the star-shaped structured surface and star-shaped for the circular structured surface (Fig. 8). These features represent the sides of the remelting tracks of the laser process as a result of different process strategies for star-shaped and circular structures, which include the boundary condition of aligning the laser-tracks non-parallel to the desired structure. Using white light interferometry, depths between $100 \mathrm{~nm}$ and $300 \mathrm{~nm}$ were measured for these undercuts.

In addition to the observed preference for adhesion at the top of the star-shaped and circular structures (Fig. 7), high resolution SE- and BSE-micrographs (Fig. 9 and Fig. 10) also suggest a preferred adhesion parallel to the undercuts as indicated by the low intensities of Al in the BSEmicrographs. However, the Al-adhesion is more severe for the star-shaped structure than for the circular one. Both, Fig. $8 \mathrm{a}$ and 9 indicate continuously build-up of $\mathrm{Al}$ in sliding direction of the Al-workpiece during tribological experiments leading to the formation of facetted Al-adhesion. Contrarily, only thin Al-adhesion spots are located on features of the circular structure (Fig. $8 \mathrm{~b}$ and 10). Moreover, the EDX-signal of the Aladhesion consists of information of both, aluminium and tool steel beneath, which is not the case for the EDXmeasurement of the star-shaped structure (Tab. 2). Thus, SEM- and EDX-investigation of the SAM-functionalized surfaces reflect the result obtained by WLI of a 2 -fold larger Al-volume adhered on the star-shaped structure compared to the circular one.

Tab. 2. Chemical composition in at. \% derived by EDX measured at spots indicated in Fig. 8.

\begin{tabular}{cccccccc} 
Pos. & $\mathrm{Al}$ & $\mathrm{Si}$ & $\mathrm{V}$ & $\mathrm{Cr}$ & $\mathrm{Mn}$ & $\mathrm{Fe}$ & $\mathrm{Mo}$ \\
\hline 1 & 99.0 & - & - & - & - & 1.0 & - \\
2 & 4.8 & 1.6 & 0.3 & 5.3 & 0.8 & 86.4 & 0.8 \\
3 & 51.1 & 0.7 & 0.3 & 3.3 & - & 44.7 & - \\
4 & - & 1.8 & 0.7 & 6.3 & - & 90.1 & 1.1
\end{tabular}

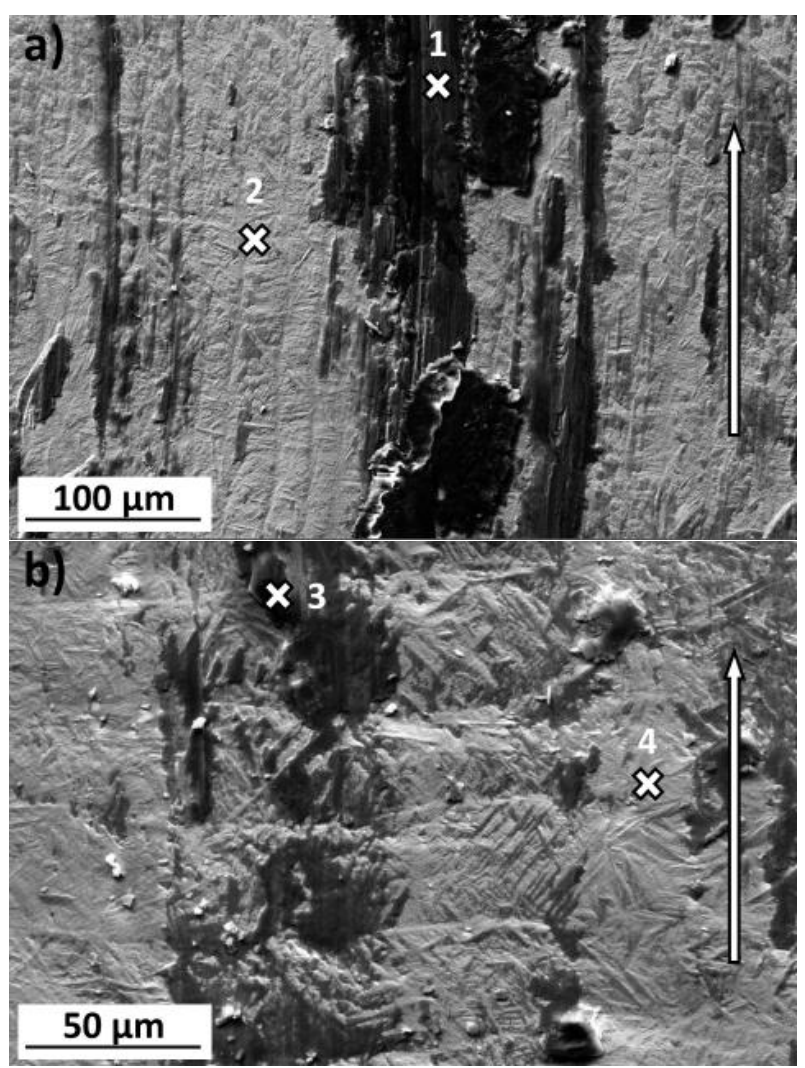

Fig. 8. SE-micrographs of laser structured surfaces after tribological tests (a) star-shaped, 250x mag. (b) circular, 500x mag. Arrow indicates the sliding direction of the Al-workpiece.

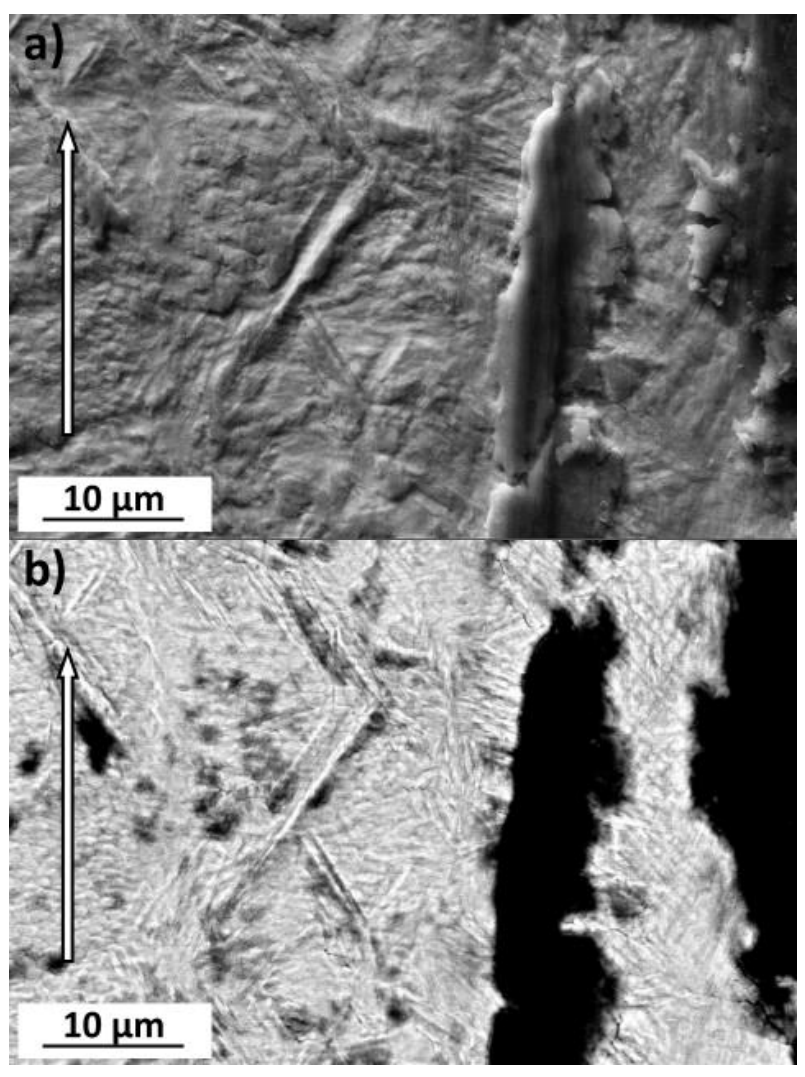

Fig. 9. High resolution (a) SE- and (b) BSE-micrograph of Al attached to a feature on the star-shaped structured surface. Arrow indicates the sliding direction of the Al-workpiece. 


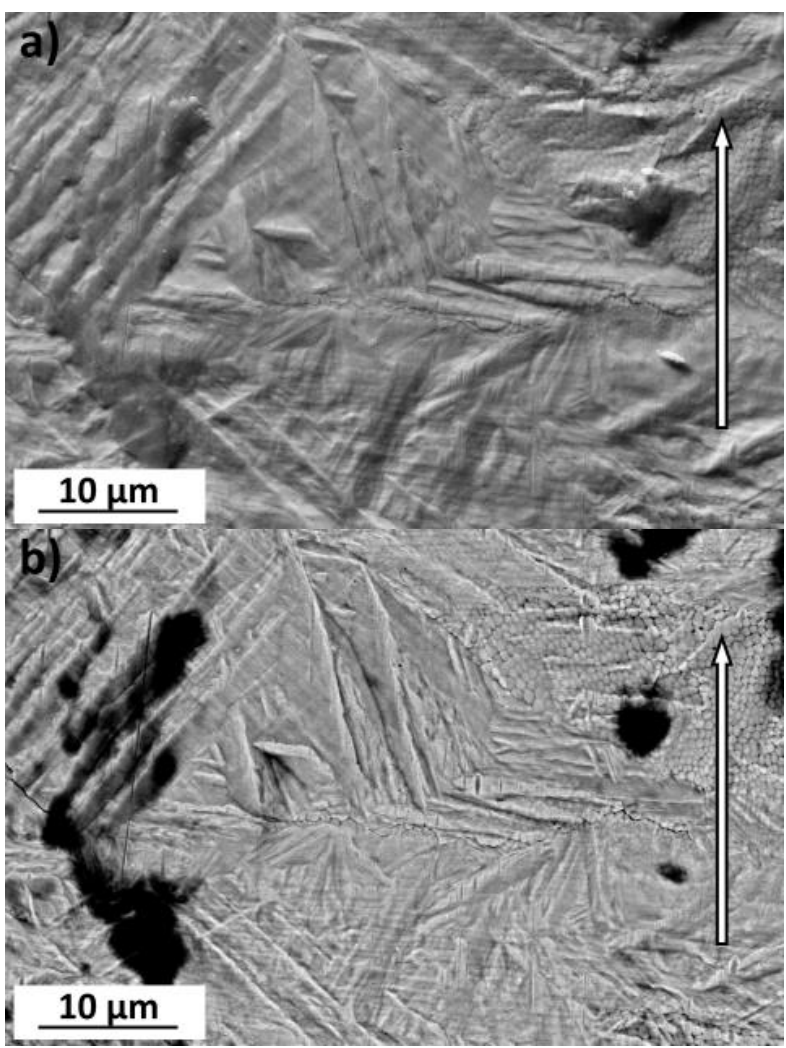

Fig. 10. High resolution (a) SE- and (b) BSE-micrograph of Al attached to a feature on the circular structured surface. Arrow indicates the sliding direction of the Al-workpiece.

\section{Conclusion and future work}

In this paper, star-shaped and circular structures with different depths were generated by laser surface processing of compression-torsion-tribometer specimens. The star-shaped structure is oriented perpendicular to the aluminium flow direction, while the circular structure is oriented parallel to the material flow direction. Besides evaluating the effect of laser structuring on the tribological behaviour, also structured tools surfaces, which were functionalized with C18PA-SAM, were tested against pure aluminium in dry conditions. The results show that both, laser structuring and surface functionalization influence the transferred torque and the amount of adhered aluminium. A structure parallel to the material flow direction is favourable, while a lower structuring depth transfers less torque. Moreover, functionalized, $1 \mu \mathrm{m}$ circular structured surfaces render a 2 -fold reduction in transferred torque and almost $75 \%$ decrease in aluminium adhesion compared to the same surface structure without the C18PA-SAM. In future work, the different mechanisms responsible for aluminium adhesion on modified surfaces will be analysed and other structural patterns will be investigated with the goals to utilize the above reported finding on geometrically complex cold forging dies.

\section{Acknowledgement}

The authors gratefully acknowledge the financial support of the Deutsche Forschungsgemeinschaft (DFG) within the priority programme SPP 1676 "Dry Metal Forming". Moreover, the authors would like to thank the Department of Ferrous Metallurgy (IEHK) of RWTH Aachen University for their support in the EDX measurements.

\section{References}

[1] F. Vollertsen, F. Schmidt, International Journal of Precision Engineering and Manufacturing-Green Technology 1 (2014) 59-62.

[2] P. Groche, C. Müller, J. Stahlmann, S. Zang, Tribology International 62 (2013) 223-231.

[3] I. Ross, A. Temmler, E. Willenborg, R. Poprawe, M. Teller, in: T. Graf, L. Overmeyer, F. Vollertsen, Lasers in Manufacturing Conference 2015, 2015.

[4] S. Prünte, D. Music, M. Teller, G. Hirt. P.H. Mutin, G. Ramanath, J.M. Schneider, J. Vac. Sci. Technol. A 35 (2017) 020605.

[5] M. Teller, S. Prünte, I. Ross, A. Temmler, J.M. Schneider, G. Hirt, AIP Conference Proceedings 1896 (2017) 140001.

[6] I. Ross, A. Temmler, M. Küpper, S. Prünte, M. Teller, J. M. Schneider, R. Poprawe, KEM 767 (2018) 69-76.

[7] N. Bay, "International Cold Forging Group - $1967-$ 1992 - Objectives, History, Published Documents", 1992.

[8] W. Zhang, N. Bay, CIRP Annals - Manufacturing Technology 41 (1992) 293-297.

[9] H. Bolvardi, D. Music, and J. M. Schneider, Thin Solid Films 589 (2015) 707-711.

[10]H. Bolvardi, D. Music, and J. M. Schneider, Appl. Surf. Sci. 332 (2015) 699-703.

[11]G. G. Fuentes, M. J. Diaz de Cerio, R. Rodriguez, J. C. Avelar-Batista, E. Spain, J. Housden, and Y. Qin, J. Mater. Process. Technol. 177 (2006) 644-648.

[12]M. Teller, M. Bambach, G. Hirt, CIRP Annals Manufacturing Technology 64 (2015) 289-292.

[13]A. Temmler, E. Willenborg, and K. Wissenbach, "Laser Polishing" Proceedings of the SPIE, Volume 8243, DOI: 10.1117/12.906001, 2012a.

[14]E. Willenborg, "Polieren von Werkzeugstählen mit Laserstrahlung" Ph.D. Thesis, RWTH Aachen, Aachen, 3-8322-4896-x, 2006.

[15]N. Pirch, S. Höges, and K. Wissenbach, "Mechanisms of surface rippling during laser polishing" Proceedings of the 8. International Seminar on Numerical Analysis of Weldability, Graz-Seggau, Austria, 2006.

[16] J. Blackburn, P. Hilton, Physics Procedia 12 (2011) 529-536.

[17]A. Temmler, “Laserumschmelzstrukturierung” PhD. Thesis, RWTH Aachen University, Aachen, ISBN 978-3-8440-1837-0, 2013.

[18]R. Ostholt, "Laserpolieren metallischer Freiformen", $\mathrm{PhD}$. Thesis, RWTH Aachen University, Aachen, ISBN 978-3-8440-0696-4, 2012.

[19]G. Guerrero, J. G. Alauzun, M. Granier, D. Laurencin, and P. H. Mutin, Dalton Trans. 42 (2013) 12569-12585. 